\title{
Produtividade de teca em sistema agroflorestal com milho e gado
}

\author{
Hudson Santos Souza ${ }^{1}$, Antonio de Arruda Tsukamoto Filho ${ }^{1}$, Daniele Aparecida Alvarenga Arriel ${ }^{2}$, Reginaldo \\ Antonio Medeiros ${ }^{3}$
}

${ }^{1}$ Universidade Federal de Mato Grosso, Rua Fernando Corrêa da Costa, 2367, Boa Esperança, Cuiabá - MT, 78068-600

${ }^{2}$ Universidade de Uberlândia, Rodovia LMG 746, Km01, s/nº , Monte Carmelo-MG, 38.500-000

${ }^{3}$ Instituto Federal de Mato Grosso, Avenida dos Ramieres, s/nº , Distrito Industrial, Cáceres - MT, 78200-000

*Autor para correspondência: hudsonssh7@gmail.com

Recebido: Setembro 2019 / Aceito: Dezembro 2019 / Publicado: Dezembro 2019

\section{Resumo}

Objetivou-se avaliar o crescimento e estimar a produtividade de teca em um sistema agroflorestal. Foram avaliados dois projetos, projeto A e projeto B. O delineamento experimental utilizado em ambos os projetos foi blocos casualizados, com quatro tratamentos e seis repetições, sendo a teca plantada com espaçamento de $4 \times 2 \mathrm{~m}$. A teca oriunda de mudas clonais obteve desenvolvimentos superiores em todos os tratamentos avaliados para as variáveis altura total, diâmetro à altura do peito, área basal, volume total e taxa de sobrevivência em todas as idades avaliadas. Até aos 72 meses de idade a presença do milho não influencia no crescimento da teca. Não houve diferença significativa em volume total entre plantar teca de material genético clonal com milho e gado em comparação com o plantio de teca de material genético clonal com milho aos 36, 48, 60 e 72 meses de idade.

Palavras-chave: Tectona grandis L.f., sustentabilidade, volumetria.

\section{Abstract}

The objective of this study was to evaluate growth and estimate teak productivity in an agroforestry system. Two projects were evaluated, project $\mathrm{A}$ and project $\mathrm{B}$. The experimental design used in both projects was randomized blocks, with four treatments and six replications, and the teak planted with $4 \times 2 \mathrm{~m}$ spacing. The teak from clonal seedlings obtained superior developments in all treatments evaluated for the variables total height, diameter at breast height, basal area, total volume and survival rate at all ages evaluated. Until 72 months of age the presence of corn does not influence the growth of teak. There was no significant difference in total volume between teak planting of clonal genetic material with maize and cattle compared to teak planting of clonal genetic material with maize at 36, 48,60 and 72 months of age.

Keywords: Tectona grandis L.f., sustainability, volumetry.

\section{Introdução}

Os sistemas agroflorestais consistem em um conjunto de técnicas que combinam intencionalmente, em uma mesma unidade de área, espécies florestais com cultivos agrícolas, com ou sem a presença de animais, para ofertar bens e serviços em base sustentáveis a partir das interações estabelecidas (Silva, 2013).

Uma das espécies madeireiras com alto valor econômico que é utilizada em sistemas agroflorestais é a Tectona grandis L.f. (Moretti et al. 2014; Khasanah et al. 2015). A teca (Tectona grandis L.f.) é uma espécie tropical decídua pertencente à família Lamiaceae que ocorre naturalmente na Índia Peninsular, Mianmar, Laos e Tailândia (Midgley et al., 2015).

De acordo com a IBÁ (2017) a área plantada com a espécie no Brasil em 2016 era de 87.502 ha. Medeiros et al.
(2015) ressalta que, mais do que aumentar a área plantada com a espécie, atualmente há uma preocupação em melhorar a produtividade das plantações, principalmente pela seleção de genótipos mais produtivos e pela adoção de sistemas de produção mais sustentáveis.

A quantificação e a previsão do crescimento dos povoamentos florestais são essenciais para se determinar a utilização dos produtos da floresta, além de fornecer informações que subsidiarão a tomada de decisão na maioria das atividades como determinação da idade ótima de corte e simulação do efeito de práticas silviculturais.

Em relação a diversas culturas florestais, pesquisas que visam estudar a quantificação e a predição do crescimento e da produção dos povoamentos de teca implantados em sistema agroflorestal ainda são escassas na literatura.

Diante disso, este trabalho foi desenvolvido com o objetivo de avaliar o crescimento e estimar a produtividade de Tectona grandis L.f. até os 72 meses de idade em sistema agroflorestal no município de Figueirópolis D’Oeste - MT.

\section{Material e Métodos \\ Localização da área do experimento}

$\mathrm{O}$ experimento foi realizado no município de Figueirópolis D'Oeste, Mato Grosso. A temperatura anual varia entre 25 a $38^{\circ} \mathrm{C}$ e as precipitações pluviométricas em torno de $1.500 \mathrm{~mm}$ ano $^{-1}$. A área experimental situa-se sob as coordenadas geográficas de latitude $15^{\circ} 24^{\prime} 27^{\prime \prime} \mathrm{S}$, longitude $58^{\circ} 45^{\prime} 56^{\prime \prime O}$ e altitude de $370 \mathrm{~m}$. O relevo é plano a suavemente ondulado. $\mathrm{O}$ solo da área foi classificado como cambissolo háplico $\mathrm{Tb}$ eutrófico léptico, segundo a classificação do Sistema Brasileiro de Classificação do Solo, apresentando textura franco-arenosa. O local onde o experimento foi instalado era ocupado anteriormente por pastagem (Brachiaria brizantha (A. Rich.) Stapf vr. Marandu).

\section{Delineamento Experimental}

$\mathrm{O}$ experimento foi composto de dois projetos: Projeto A e Projeto B.

O delineamento experimental utilizado para o Projeto A foi blocos casualizados, com quatro tratamentos e seis repetições (Tabela 1). O primeiro fator foi o tipo de propagação de muda (seminal e clonal). As mudas clonais de teca foram fornecidas por uma empresa reflorestadora e as mudas seminais adquiridas de produtores de mudas da região. $\mathrm{O}$ segundo fator foi o milho nas entrelinhas da teca, em duas situações (presença e ausência), plantados aos 12 e 24 meses.

Como a área foi ocupada antes pela pastagem (Brachiaria brizantha (A. Rich.) Stapf vr. Marandu), houve uma reinfestação não precisando realizar a semeadura da pastagem. 
Tabela 1. Identificação dos quatro tratamentos do Projeto A.

\begin{tabular}{|c|c|c|}
\hline Trat. & Sistemas & Práticas \\
\hline 1 & Agrossilvipastoril & teca clonal com milho e gado \\
\hline 2 & Silvipastoril & teca clonal com gado \\
\hline 3 & Agrossilvipastoril & teca seminal com milho e gado \\
\hline 4 & Silvipastoril & teca seminal com milho gado \\
\hline
\end{tabular}

Em todos os tratamentos do Projeto A, aos 36 meses, foi inserido o gado da raça Girolando, e bovinos da raça Gir, sendo uma raça adaptada ao clima tropical e adequada para a produção de leite. A quantidade de bovinos colocada na área foi de duas Unidade Animal ha ${ }^{-1}$.

O delineamento experimental utilizado para o Projeto B foi blocos casualizados, com quatro tratamentos e seis

Tabela 2. Identificação dos quatro tratamentos do Projeto B.

\begin{tabular}{|c|c|c|}
\hline Trat. & Sistemas & Práticas \\
\hline 1 & Agrossilvicultural & teca clonal com milho \\
\hline 2 & Monocultivo & teca clonal \\
\hline 3 & Agrossilvicultural & teca seminal com milho \\
\hline 4 & Monocultivo & teca seminal \\
\hline
\end{tabular}

Como a área foi ocupada antes pela pastagem, houve uma reinfestação dela. Aos 36 meses, a pastagem enfestada no projeto B foi eliminada com aplicação de herbicida.

Para ambos projetos, cada parcela possui sete linhas de plantio de teca no sentido leste-oeste, com seis plantas por linha, no espaçamento $4 \times 2 \mathrm{~m}$.

\section{Coleta de dados}

A coleta de dados do inventário florestal foi realizada aos 36, 48, 60 e 72 meses após a implantação do experimento. Foram coletados os dados de altura total $(h t)$ das árvores que perfaziam a linha central de cada parcela (5 árvores) e circunferência à $1,30 \mathrm{~m}$ do solo (cap) de todas as 20 árvores na área útil da parcela.

Foram realizadas as avaliações da porcentagem de sobrevivência $(S \%)$, diâmetro à altura de $1,30 \mathrm{~m}($ dap $)$, altura total $(h t)$, área basal $(G)$, volume total $(v t)$, crescimento, produtividade, incremento corrente anual (ica) e incremento médio anual (ima).

A porcentagem de sobrevivência $(S \%)$ foi calculada com base na contagem das plantas vivas e estabelecimento da proporção em relação ao número total de plantas da área útil da parcela (20 árvores). Para a análise estatística, os dados de sobrevivência dos blocos foram transformados para arcoseno $\sqrt{x / 100}$, a fim de atender as preposições da análise de variância (ANOVA).

Foram coletados os dados de circunferência a $1,30 \mathrm{~m}$ do solo (dap) de todas árvores com o auxílio de fita métrica. Com os dados de cap, foram determinados os dap por árvore, dividindo-se cada valor de cap por $\pi(3,14)$.

A altura total $(h t)$ foi coletada somente das árvores que perfaziam a linha central de cada parcela (5 árvores). Para estimar a $h t$ das árvores restantes, foi ajustado um modelo de relação hipsométrica por cada tratamento:

$$
\text { Ln } h t=\beta_{0}+\beta_{1}(1 / \text { dap })+\beta_{2}(1 / \mathrm{I})+\mathrm{e}
$$

Em que:

$\mathrm{Ln}=$ Logaritmo neperiano;

$h t=$ Altura total da árvore $(\mathrm{m})$;

dap $=$ Diâmetro à $1,30 \mathrm{~m}(\mathrm{~cm})$;

$\mathrm{I}=$ Idade $(\operatorname{anos})$

$\beta_{\mathrm{i}}=$ Parâmetros dos modelos;

$\mathrm{e}=$ Erro da estimativa.

A área seccional $\left(g_{\mathrm{i}}\right)$ do indivíduo arbóreo foi calculada pela fórmula: $g_{\mathrm{i}}=\frac{\pi \cdot d a p^{2}}{40000}$ repetições (Tabela 2). O primeiro fator foi o tipo de propagação de muda (seminal e clonal). O segundo fator foi o milho nas entrelinhas da teca, em duas situações (presença e ausência), plantado aos 12 e 24 meses. No projeto B não foi inserido o gado. $\sum_{\mathrm{i}=1}^{\mathrm{n}} g_{i}$

A área basal por bloco (G.bl) pela fórmula: $G . \mathrm{bl}=$

Posteriormente foi feita a conversão para a Área Basal por hectare $(G): G=\frac{G \cdot b l \cdot 10000}{160}$

Em que:

$g_{i}=$ Área seccional $\left(\mathrm{m}^{2}\right)$;

$G . b l=$ Área basal por bloco $\left(\mathrm{m}^{2} \mathrm{bl}^{-1}\right)$;

$G=$ Área basal por hectare $\left(\mathrm{m}^{2} \mathrm{ha}^{-1}\right)$;

dap = Diâmetro à $1,30 \mathrm{~m}(\mathrm{~cm})$;

$\pi=$ Constante 3,141592654 .

Para a estimativa do volume individual das árvores, foi aplicado a equação de Schumacher e Hall ajustado por Cruz et al. (2008):

$\operatorname{Ln}\left(v_{i}\right)=-8,57046+1,07588 \operatorname{Ln}($ dap $)+$

$1,27429 \operatorname{Ln}(h t) \quad \mathrm{R}^{2} 0,987$

Em que:

Ln = Logaritmo neperiano;

$v_{\mathrm{i}}=$ Volume individual $\left(\mathrm{m}^{3}\right)$;

$h t=$ Altura total da árvore $(\mathrm{m})$

dap = Diâmetro à $1,30 \mathrm{~m}(\mathrm{~cm})$.

O volume total por bloco ( $v t$.bl) foi calculado por meio da soma dos volumes das árvores nos blocos e posteriormente convertido para volume total por hectare $(v t)$ em metros cúbicos $\left(\mathrm{m}^{3}\right)$.

Para avaliar o crescimento das variáveis dap $(\mathrm{cm}), h t(\mathrm{~m})$ e produtividade das variáveis $G\left(\mathrm{~m}^{2} \mathrm{ha}^{-1}\right)$ e $v t\left(\mathrm{~m}^{3} \mathrm{ha}^{-1}\right)$ foi utilizado o modelo de Schumacher, amplamente utilizado nos estudos de crescimento florestal (Campos e Leite, 2013):

Em que:

$$
\mathrm{Y}=\mathrm{e}^{\beta_{0}+\beta_{1}\left(\frac{1}{\mathrm{I}}\right)}+\mathrm{e}
$$

$\mathrm{Y}=$ Crescimento em uma idade;

$\mathrm{I}=$ Idade $(\operatorname{anos})$;

$\beta_{\mathrm{i}}=$ Parâmetros dos modelos

$\mathrm{e}=$ Erro da estimativa

O incremento corrente anual (ica) das variáveis altura total $(h t)$, diâmetro à altura do peito $($ dap $)$, área basal $(G) \mathrm{e}$ volume total $(v t)$, foi calculado pela diferença de crescimento entre as avaliações no período de um ano e o incremento médio anual (ima) das variáveis foi calculado pela divisão do crescimento pela idade atual do povoamento florestal, em anos, por ocasião de cada avaliação. 
Os resultados obtidos foram submetidos à análise de variância, após terem sido atendidos os requisitos de normalidade Shapiro-Wilks e homogeneidade das variâncias Bartlett. Os valores médios foram comparados entre si pelo teste de Tukey a 5\% de probabilidade.

\section{Resultados}

Projeto A

$\mathrm{Na}$ Tabela 3 encontram-se os resultados das análises de taxa de sobrevivência $(S)$, diâmetro à altura do peito (dap), altura total $(h t)$, área basal $(G)$ e volume total $(v t)$ de teca para os quatro tratamentos aos $36,48,60$ e 72 meses de idade no projeto A.

As equações para a estimativa do crescimento e produtividade do Projeto A foram ajustadas com os dados coletados aos 36, 48, 60 e 72 meses. Nas Figuras com as curvas de incremento corrente anual (ica) e incremento médio anual (ima), como o crescimento é o parâmetro estimado em função da idade, foi estimado o crescimento nas idades anteriores 12 e 24 meses para melhor visualização da tendência das curvas.

\section{Projeto $B$}

$\mathrm{Na}$ Tabela 4 encontram-se os resultados das análises de taxa de sobrevivência $(S)$, diâmetro à altura do peito (dap), altura total $(h t)$, área basal $(G)$ e volume total $(v t)$ de teca para os quatro tratamentos aos $36,48,60$ e 72 meses no projeto B.

$\mathrm{O}$ ajuste das equações para a estimativa do crescimento e produtividade do Projeto B foi realizado com os dados coletados aos 36, 48, 60 e 72 meses. Nas Figuras com as curvas de incremento corrente anual (ica) e incremento médio anual (ima), como o crescimento é o parâmetro estimado em função da idade, foi estimado o crescimento nas idades anteriores 12 e 24 meses para uma visualização melhor da tendência das curvas.

Tabela 3. Taxa de sobrevivência $(S)$, Diâmetro à altura do peito (dap), Altura total $(h t)$, Área basal $(G)$ e Volume total $(v t)$ de teca para os quatro tratamentos no Projeto A.

\begin{tabular}{|c|c|c|c|c|}
\hline \multirow{3}{*}{ Trat. } & \multicolumn{4}{|c|}{ Idade (meses) } \\
\hline & 36 & 48 & 60 & 72 \\
\hline & \multicolumn{4}{|l|}{$S(\%)$} \\
\hline 1 & $99,2 \mathrm{ab}$ & $98,3 \mathrm{a}$ & $97,5 \mathrm{a}$ & $97,5 \mathrm{a}$ \\
\hline 2 & $100,0 \mathrm{a}$ & $100,0 \mathrm{a}$ & $100,0 \mathrm{a}$ & $100,0 \mathrm{a}$ \\
\hline 3 & $90,0 \mathrm{~b}$ & $88,3 \mathrm{~b}$ & $87,5 \mathrm{~b}$ & $85,8 \mathrm{~b}$ \\
\hline \multirow[t]{2}{*}{4} & $93,3 \mathrm{ab}$ & $92,5 a b$ & $92,5 \mathrm{ab}$ & $92,5 \mathrm{ab}$ \\
\hline & \multicolumn{4}{|c|}{ dap $(\mathrm{cm})$} \\
\hline 1 & 7,53 a & 9,58 a & 11,86 a & 13,06 a \\
\hline 2 & 7,89 a & 9,80 a & $12,05 \mathrm{a}$ & 13,38 a \\
\hline 3 & $6,00 \mathrm{~b}$ & $6,83 \mathrm{~b}$ & $7,60 \mathrm{~b}$ & $8,54 \mathrm{~b}$ \\
\hline \multirow[t]{2}{*}{4} & $6,01 \mathrm{~b}$ & $7,02 \quad b$ & $7,60 \mathrm{~b}$ & $8,47 \quad b$ \\
\hline & \multicolumn{4}{|l|}{$h t(\mathrm{~m})$} \\
\hline 1 & $7,69 \mathrm{a}$ & 9,98 a & $11,72 \mathrm{a}$ & $12,65 \mathrm{~b}$ \\
\hline 2 & $8,05 \mathrm{a}$ & $10,10 \mathrm{a}$ & $12,10 \mathrm{a}$ & $13,39 \mathrm{a}$ \\
\hline 3 & $6,73 \mathrm{~b}$ & 7,79 b & $8,66 \mathrm{~b}$ & $8,87 \mathrm{c}$ \\
\hline \multirow[t]{2}{*}{4} & $6,55 \mathrm{~b}$ & $7,52 \mathrm{~b}$ & $8,56 \mathrm{~b}$ & $8,80 \mathrm{c}$ \\
\hline & \multicolumn{4}{|c|}{$G\left(\mathrm{~m}^{2} \mathrm{ha}^{1}\right)$} \\
\hline 1 & $5,52 \mathrm{a}$ & $8,90 \mathrm{a}$ & $13,46 \mathrm{a}$ & $16,37 \mathrm{a}$ \\
\hline 2 & $6,25 \mathrm{a}$ & $9,67 \mathrm{a}$ & $14,50 \mathrm{a}$ & $17,85 \mathrm{a}$ \\
\hline 3 & $3,20 \mathrm{~b}$ & $4,08 \mathrm{~b}$ & $5,05 \mathrm{~b}$ & $6,28 \mathrm{~b}$ \\
\hline \multirow[t]{2}{*}{4} & $3,41 \mathrm{~b}$ & $4,58 \mathrm{~b}$ & $5,52 \mathrm{~b}$ & $6,78 \mathrm{~b}$ \\
\hline & \multicolumn{4}{|c|}{$v t\left(\mathrm{~m}^{3} \mathrm{ha}^{-1}\right)$} \\
\hline 1 & $27,71 \mathrm{a}$ & $50,30 \mathrm{a}$ & 76,03 a & 93,78 a \\
\hline 2 & $31,45 \mathrm{a}$ & $54,00 \mathrm{a}$ & $83,32 \mathrm{a}$ & $107,09 \mathrm{a}$ \\
\hline 3 & $16,50 \mathrm{~b}$ & $22,62 \mathrm{~b}$ & $29,59 \mathrm{~b}$ & $33,45 \mathrm{~b}$ \\
\hline 4 & $17,45 \mathrm{~b}$ & $24,42 \mathrm{~b}$ & $31,74 \mathrm{~b}$ & $36,10 \mathrm{~b}$ \\
\hline
\end{tabular}

Tabela 4. Taxa de sobrevivência $(S)$, Diâmetro à altura do peito (dap), Altura total $(h t)$, Área basal $(G)$ e Volume total $(v t)$ de teca para os quatro tratamentos no Projeto B.

\begin{tabular}{|c|c|c|c|c|}
\hline \multirow{3}{*}{ Trat. } & \multicolumn{4}{|c|}{ Idade (meses) } \\
\hline & 36 & 48 & 60 & 72 \\
\hline & \multicolumn{4}{|l|}{$S(\%)$} \\
\hline 1 & $100 \mathrm{a}$ & $100 \mathrm{a}$ & $100 \mathrm{a}$ & $97,5 \mathrm{a}$ \\
\hline 2 & $98,5 \mathrm{a}$ & $98,5 \mathrm{ab}$ & 98,3 a & $95,0 \mathrm{a}$ \\
\hline 3 & $96,8 \mathrm{ab}$ & $96,7 \mathrm{ab}$ & $96,7 \mathrm{ab}$ & $95,0 \mathrm{a}$ \\
\hline \multirow[t]{2}{*}{4} & $90,8 \mathrm{~b}$ & $90,8 \mathrm{~b}$ & $90,8 \mathrm{~b}$ & $90,0 \mathrm{a}$ \\
\hline & \multicolumn{4}{|c|}{ dap $(\mathrm{cm})$} \\
\hline 1 & 8,00 a & $11,76 a$ & $13,74 \mathrm{a}$ & $15,27 \mathrm{a}$ \\
\hline 2 & $7,78 \mathrm{a}$ & $11,43 \mathrm{a}$ & $13,80 \mathrm{a}$ & $15,39 \mathrm{a}$ \\
\hline 3 & $6,64 b$ & $8,52 \mathrm{~b}$ & $10,19 \mathrm{~b}$ & $11,14 \mathrm{~b}$ \\
\hline \multirow[t]{2}{*}{4} & $6,22 \mathrm{~b}$ & $8,28 \mathrm{~b}$ & $10,11 \mathrm{~b}$ & $11,04 \mathrm{~b}$ \\
\hline & \multicolumn{4}{|l|}{$h t(\mathrm{~m})$} \\
\hline 1 & $8,16 \mathrm{a}$ & $11,04 \mathrm{a}$ & $12,70 \mathrm{a}$ & $14,14 \mathrm{a}$ \\
\hline 2 & $7,98 \mathrm{a}$ & $10,73 \mathrm{a}$ & $12,62 \mathrm{a}$ & $13,85 \mathrm{a}$ \\
\hline 3 & $7,28 \mathrm{~b}$ & $8,87 \mathrm{~b}$ & $10,10 \mathrm{~b}$ & $11,20 \mathrm{~b}$ \\
\hline \multirow[t]{2}{*}{4} & $6,88 \mathrm{~b}$ & $8,58 \mathrm{~b}$ & $9,75 \mathrm{c}$ & $10,86 \mathrm{c}$ \\
\hline & \multicolumn{4}{|c|}{$G\left(\mathrm{~m}^{2} \mathrm{ha}^{-1}\right)$} \\
\hline 1 & $6,34 \mathrm{a}$ & $13,67 \mathrm{a}$ & $18,66 \mathrm{a}$ & $22,48 \mathrm{a}$ \\
\hline 2 & $5,85 \mathrm{a}$ & $12,62 \mathrm{a}$ & $18,55 \mathrm{a}$ & $22,23 \mathrm{a}$ \\
\hline 3 & $4,22 \mathrm{~b}$ & $7,02 \mathrm{~b}$ & $10,04 \mathrm{~b}$ & $11,78 \mathrm{~b}$ \\
\hline \multirow[t]{2}{*}{4} & $3,57 \mathrm{~b}$ & $6,24 \mathrm{~b}$ & $9,33 \mathrm{~b}$ & $11,03 \mathrm{~b}$ \\
\hline & \multicolumn{4}{|c|}{$v t\left(\mathrm{~m}^{3} \mathrm{ha}^{-1}\right)$} \\
\hline 1 & $32,47 \mathrm{a}$ & $72,07 a$ & $101,65 a$ & $127,41 \mathrm{a}$ \\
\hline 2 & 29,89 a & $65,87 \mathrm{a}$ & 99,78 a & $121,88 \mathrm{a}$ \\
\hline 3 & $22,25 \mathrm{~b}$ & $37,75 \mathrm{~b}$ & $53,68 \mathrm{~b}$ & $66,16 \mathrm{~b}$ \\
\hline 4 & $18,75 \mathrm{~b}$ & $33,27 \mathrm{~b}$ & $48,23 \mathrm{~b}$ & $60,14 \mathrm{~b}$ \\
\hline
\end{tabular}

\section{Discussão}

Projeto A

Aos 48, 60 e 72 meses, as taxas de sobrevivência ( $S \%)$ dos tratamentos 1 e 2 (Tabela 3) foram as maiores e não tiveram diferença significativa entre elas. Já para o tratamento quatro a taxa de sobrevivência foi de $92,5 \%$ e para o tratamento 3 foi $85,8 \%$ aos 72 meses.

Os tratamentos 1 e 2 apresentaram as maiores médias para o diâmetro à altura do peito e não houve diferença significativa entre eles (Tabela 3). Os tratamentos 3 e 4 também não diferiram entre si, mas foram estatisticamente inferiores aos tratamentos com plantas clonais.

Verificou-se que a partir dos 36 meses de idade, já não houve diferença de crescimento em diâmetro com o fator milho presente nos dois primeiros anos do plantio. Isso demonstrou que pode ser mais vantajoso do ponto de vista econômico o plantio de teca com milho, em virtude de uma renda a mais com a cultura agrícola. Pode-se observar que as plantas clonais de teca (tratamentos 1 e 2) foram em média $36 \%$ maiores em dap do que as plantas seminais (tratamentos 3 e 4).

Resultados semelhantes foram encontrados por Silva et al. (2014), onde os autores avaliaram o desempenho inicial de Tectona grandis em sistema silvipastoril na região norte de Mato Grosso em uma área composta por pastagem tipo Brachiaria brizantha implantada sob espaçamento $25 \times 3 \mathrm{~m}$. Aos 53 meses de idade da teca o dap médio das árvores foi $10,83 \mathrm{~cm}$.

Os tratamentos 1 e 2 tiveram médias de altura total superiores em relação as demais aos 36, 48 e 60 meses (Tabela 3). Aos 72 meses o tratamento 2 se destacou dos demais apresentando a maior média de $h t(13,39 \mathrm{~m})$. Os tratamentos 3 e 4 apresentaram as menores médias em $h t$ não apresentando diferença significativa entre eles. As árvores dos tratamentos com clones superiores (1 e 2) foram em média $32 \%$ maiores que dos tratamentos seminais ( 3 e 4 ). 
De acordo com Xavier e Silva (2010) a seleção clonal há uma tendência de se desenvolver materiais mais específicos tanto para uma maior adaptação a diferentes condições edafoclimáticas, quanto para qualidades diversas da madeira. A adoção de clones específicos será cada vez mais implementada, principalmente em função dos avanços que da silvicultura de precisão, permitindo a definição do manejo mais apropriado a um determinado clone. Os clones produzidos especialmente para um produto trarão ganhos na qualidade e na redução de custos inerentes a utilização de uma matéria prima mais indicada para a obtenção do produto final.

Os tratamentos 1 e 2 apresentaram as maiores médias em área basal $(G)$ não diferindo entre si (Tabela 3). Já os tratamentos 3 e 4 apresentaram as menores médias. Os tratamentos que foram implantadas árvores clonais de teca (1 e 2) tiveram uma área basal em média de $62 \%$ maiores que os tratamentos seminais $(3$ e 4$)$. Os tratamentos 1 e 2 apresentaram valores de área basal superiores aos encontrado por Tonini et al. (2009) em Iracema - Roraima, onde, aos 78 meses, o plantio homogêneo com Tectona grandis apresentou uma área basal de $12,9 \mathrm{~m}^{2} \mathrm{ha}^{-1}$.

Os tratamentos 1 e 2 apresentaram as maiores médias de volumes totais ( $v t)$ não diferindo entre si (Tabela 3 ). Já os tratamentos 3 e 4 apresentaram as menores médias. Foi observada uma acentuada diferença no volume entre os tratamentos clonais e seminais, em que os tratamentos clonais (1 e 2) tiveram um volume $65 \%$ maior que que os tratamentos seminais (3 e 4).

De acordo com Medeiros et al. (2015), clones superiores apresentaram maior crescimento e produção em relação às seminais, independentemente do sistema de cultivo. Esses resultados corroboram com informações de empresas reflorestadoras no Estado de Mato Grosso, em que mudas de teca oriundas de clones selecionados apresentam ganhos de produtividade nas idades iniciais de até $36 \%$.

Segundo Xavier et al. (2009) o material genético clonal por manter as características genéticas da planta mãe, apresenta maior uniformidade no povoamento e possuir uma melhor adaptação às características do sítio. Medeiros et al. (2015), ressalta que atualmente, esses ganhos de produtividade e a demanda de madeira em qualidade e quantidade exigidas pelo mercado têm elevado o interesse por clones de teca mais produtivos.

Plantar milho nos estágios iniciais de crescimento de teca fornece uma vantagem em relação à monocultura de teca, independentemente do espaçamento utilizado (Santos-M artin e Van Noordwijk, 2009; Khasanah et al. 2015).

Os ajustes para estimativa do crescimento apresentaram coeficientes de regressão e valores de $\mathrm{F}$ significativos a $5 \%$ de probabilidade. Os ajustes forneceram valores de erro padrão residual $(\operatorname{Syx})$ menores que 0,19 . O coeficiente de determinação $\left(\mathrm{R}^{2}\right)$ também se apresentou satisfatório com valores acima de 0,71 .

$\mathrm{O}$ crescimento em diâmetro dos tratamentos 1 e 2 foram semelhantes não diferindo estatisticamente entre si aos 36,48 , 60 e 72 meses (Figura 1). Já os tratamentos 3 e 4 tiveram os menores crescimento. Pode-se observar uma nítida diferença no crescimento entre os tratamentos clonais ( 1 e 2 ) e seminais (3 e 4). Essa diferença, pode ser explicada pela qualidade inferior do material genético seminal usado no plantio.
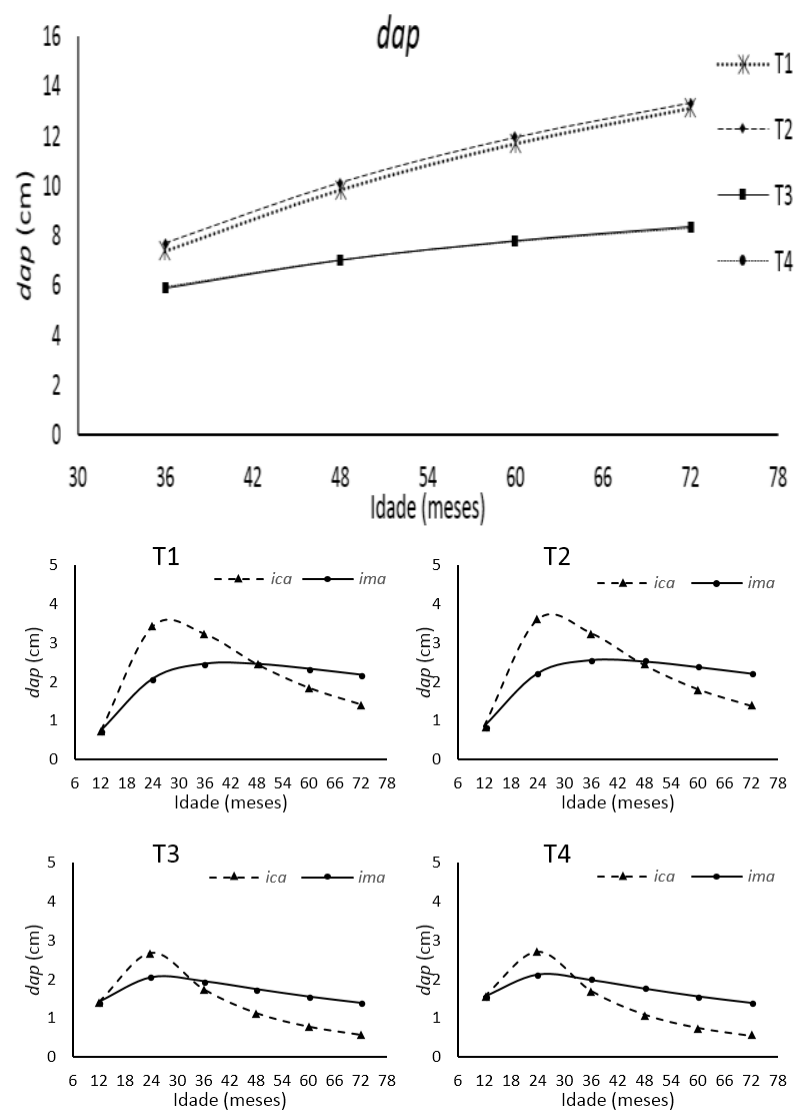

Figura 1. Curvas de crescimento e curvas de incremento corrente anual (ica) e incremento médio anual (ima) em diâmetro à altura de 1,30 m (dap) $(\mathrm{cm})$ de teca para os quatro tratamentos no projeto $\mathrm{A}$.

Para a altura total $(h t)$, o tratamento 2 obteve o maior crescimento, seguido do tratamento 1 (Figura 2). Os tratamentos 3 e 4 tiveram os menores crescimento em altura, não tendo diferença significativa entre si.

$\mathrm{O}$ crescimento em volume total dos tratamentos 1 e 2 foram semelhantes não diferindo estatisticamente entre si aos 72 meses (Figura 4). Os tratamentos 3 e 4 tiveram os menores crescimento, possuindo uma leve tendência em estabilização da curva. Nota-se uma acentuada diferença no crescimento em volume entre os tratamentos clonais (1 e 2) e seminais (3 e 4).

A teca de clone superior com milho e gado foi tão produtiva em volume total quanto à teca com milho sem gado. Neste sentido em relação à produtividade, ambos, são iguais obtendo vantagem do ponto de vista econômico em relação a um plantio em monocultivo.

Para a área basal $(G)$, os tratamentos 1 e 2 apresentaram os maiores crescimentos (Figura 3). Os tratamentos 3 e 4 tiveram os menores crescimento em área basal, não tendo diferença significativa. 

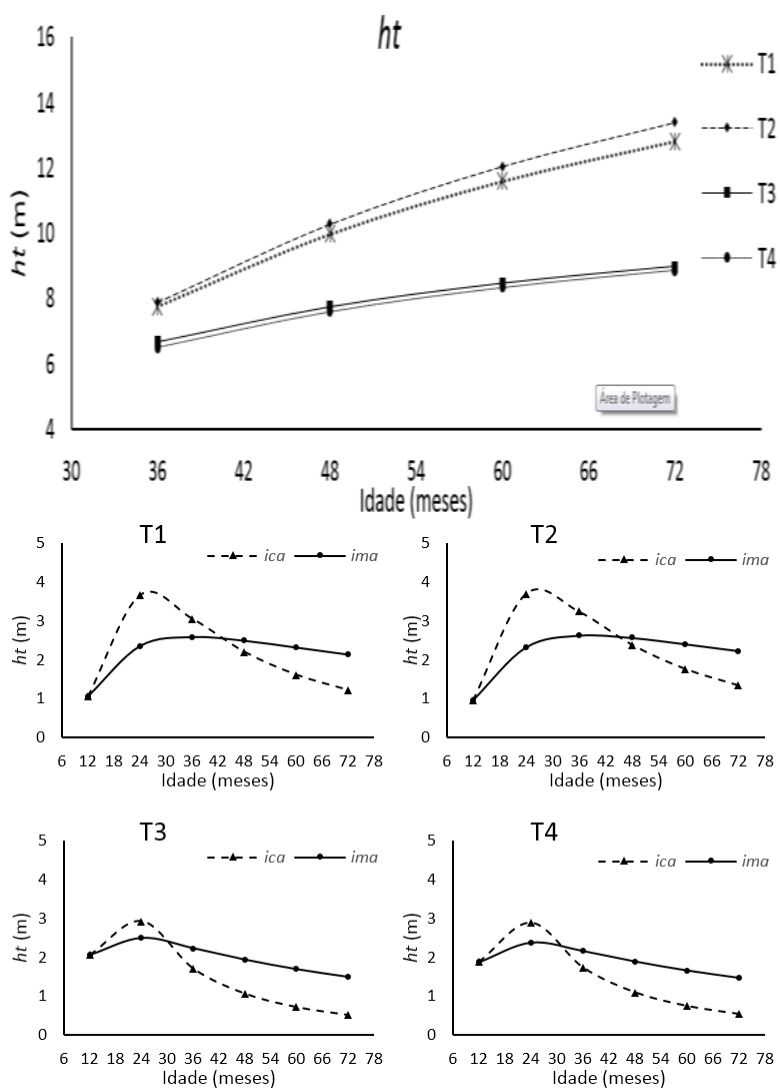

Figura 2. Curvas de crescimento e curvas de incremento corrente anual (ica) e incremento médio anual (ima) em altura total $(h t)(\mathrm{m})$ de teca para os quatro tratamentos no projeto $\mathrm{A}$.
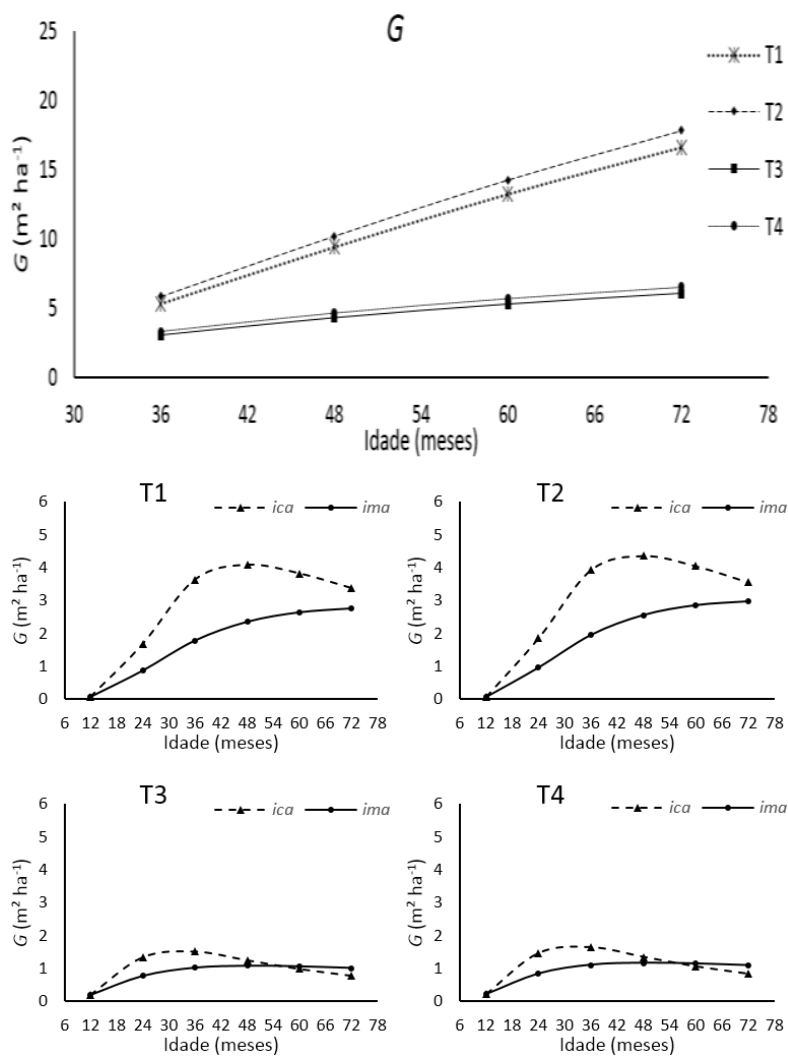

Figura 3. Curvas de crescimento e curvas de incremento corrente anual (ica) e incremento médio anual (ima) em área basal $(\mathrm{G})\left(\mathrm{m}^{2} \mathrm{ha}^{-1}\right)$ de teca para os quatro tratamentos no projeto A.
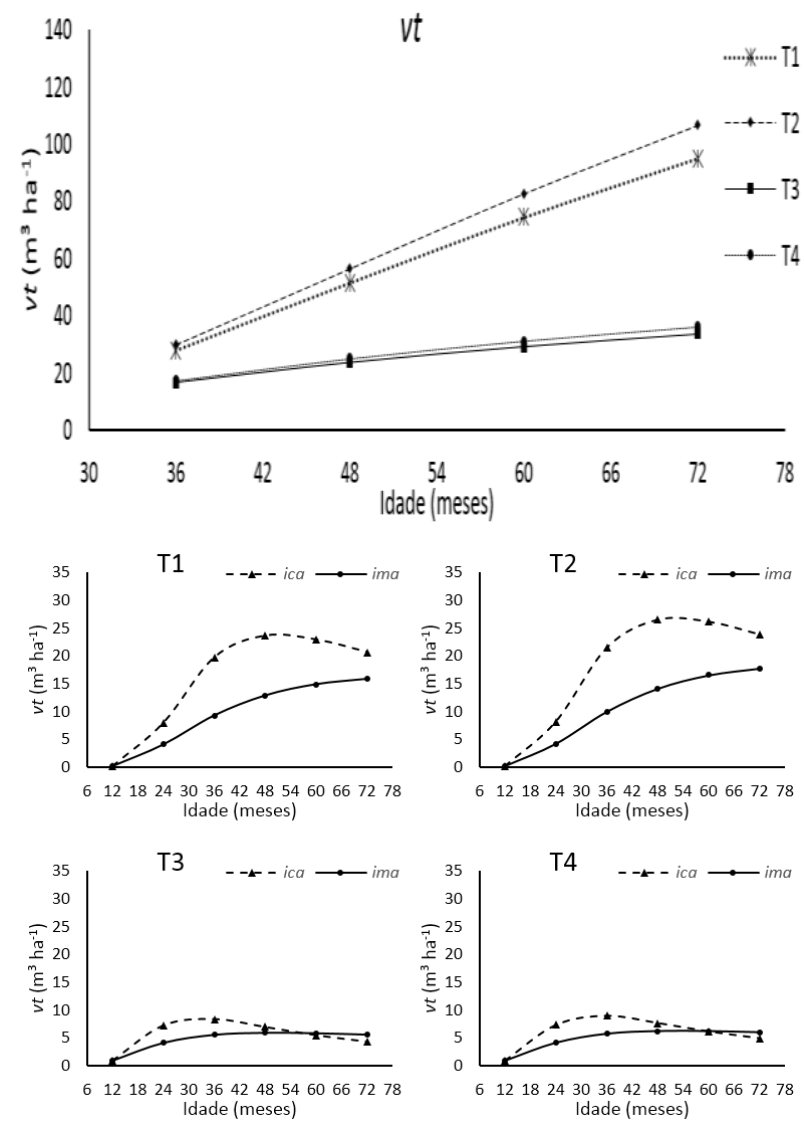

Figura 4. Curvas de crescimento e curvas de incremento corrente anual (ica) e incremento médio anual (ima) em volume total $(v t)\left(\mathrm{m}^{3} \mathrm{ha}^{-1}\right)$ de teca no projeto A.

\section{Projeto $B$}

Aos 36 meses, os tratamentos 1, 2 e 3 não apresentaram diferença significativa para a sobrevivência, entretanto, foi verificado que o tratamento 4 , diferiu dos tratamentos 1 e 2 , aos 36, 48 e 60 meses, sendo inferior (Tabela 4). Pode-se observar que os tratamentos $1 \mathrm{com}$ teca clonal e 2 com teca seminal obtiveram vantagem na taxa de sobrevivência em relação aos tratamentos de monocultura da teca até os 60 meses.

Entretanto, aos 72 meses, foi verificado que não houve mais diferença significativa entre as taxas de sobrevivência dos tratamentos. Assim, de maneira geral, os quatro tratamentos tiveram uma taxa de sobrevivência satisfatória.

As taxas de sobrevivência deste trabalho foram superiores ao encontrado por Silva et al. (2016) em povoamento de teca em monocultivo implantado em Cáceres - MT, onde a sobrevivência das árvores aos 11 anos foi de 90\%. As taxas de sobrevivência deste trabalho também foram superiores às taxas encontradas por Ribeiro et al. (2006) em Minas Gerais, em diferentes níveis de adubação e com sobrevivência entre $63,8 \%$ e $90,0 \%$.

Os tratamentos 1 e 2 apresentaram os maiores diâmetros à altura do peito aos 36, 48, 60 e 72 meses e não houve diferença significativa entre eles (Tabela 4). Os tratamentos 3 e 4 também não diferiram entre si, porém foram inferiores aos tratamentos com clones. Nota-se que os tratamentos com clones superiores foram em média $28 \%$ maiores em dap em relação aos tratamentos seminais.

Os tratamentos 1 e 2 (plantas clonais) apresentaram as maiores médias para a altura total e não houve diferença significativa entre eles. Os tratamentos 3 e 4 também não diferiram entre si, mas foram estatisticamente inferiores aos 
tratamentos com plantas clonais (Tabela 4). Independente do tratamento, plantas clonais foram $21 \%$ mais altas que as plantas seminais.

Os tratamentos 1 e 2 apresentaram altura total superior aos 3 e 4, contudo entre os primeiros assim como os segundos não houve diferença significativa entre si. Independente do tratamento, plantas clonais foram $21 \%$ mais altas que as plantas seminais (Tabela 4).

Os tratamentos 1 e 2 apresentaram as maiores médias em área basal $(G)$ não diferindo entre si. Já os tratamentos 3 e 4 apresentaram média inferiores aos tratamentos 1 e 2 . Os tratamentos com árvores de clones superiores (1 e 2) tiveram uma área basal em média de $49 \%$ maiores que os tratamentos seminais (3 e 4) (Tabela 4).

$\mathrm{O}$ volume total dos tratamentos 1 e 2 foram os maiores do que os tratamentos 3 e 4, não diferindo entre si estatisticamente. Não houve diferença significativa para os tratamentos 3 e 4 que foram inferiores (Tabela 4). Observouse uma significativa diferença no volume entre os tratamentos com clones superiores e seminais, em que os tratamentos com clones superiores ( 1 e 2) tiveram um volume $49 \%$ maior que os tratamentos seminais (3 e 4 ).

Os ajustes forneceram valores de erro padrão residual (Syx) menores que 0,17 . O coeficiente de determinação $\left(R^{2}\right)$ também se apresentou satisfatório com valores acima de 0,70 .

O crescimento em diâmetro da teca nos tratamentos 1 e 2 foram semelhantes (Figura 5) não diferindo estatisticamente entre si aos 72 meses e superiores aos tratamentos 3 e 4 . Os tratamentos 3 e 4 tiveram os menores crescimento. Houve uma notável diferença no crescimento entre os tratamentos clonais ( 1 e 2 ) e seminais (3 e 4$)$.
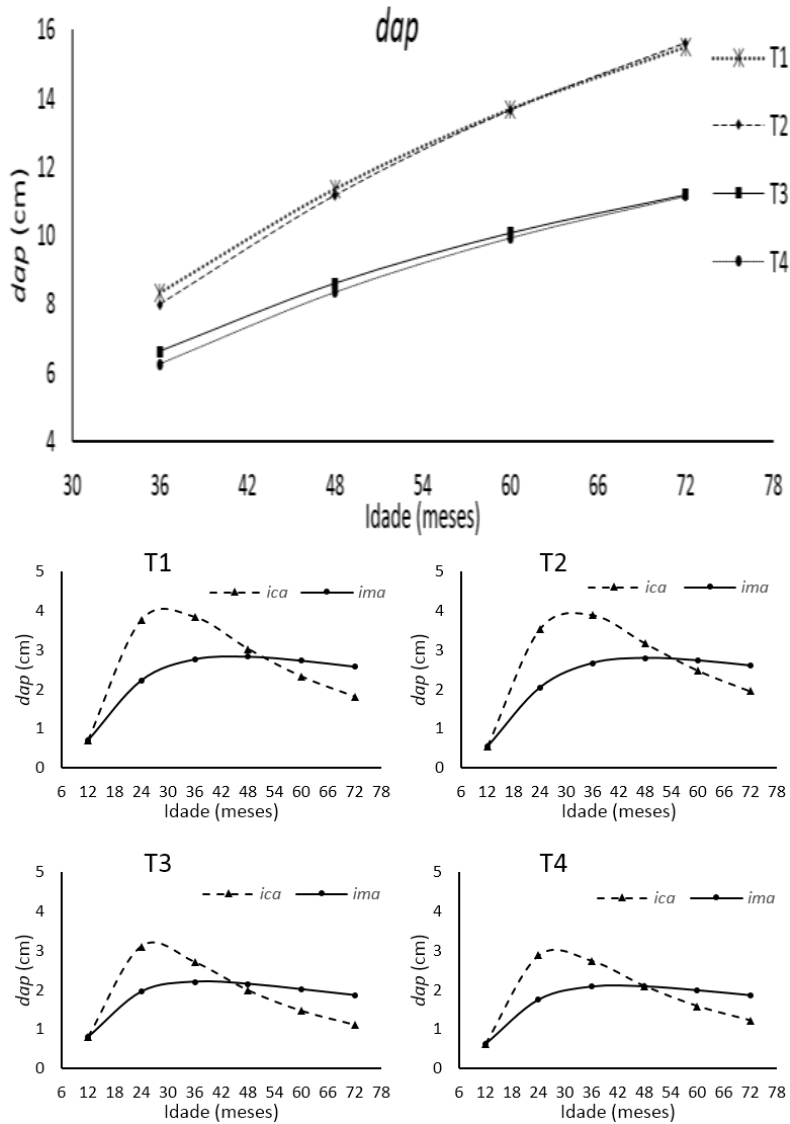

Figura 5. Curvas de crescimento e curvas de incremento corrente anual (ica) e incremento médio anual (ima) em diâmetro à altura do peito $($ dap $)(\mathrm{cm})$ de teca para os quatro tratamentos no projeto $\mathrm{B}$.
Para a altura total $(h t)$, os tratamentos 1 e 2 obtiveram os maiores crescimentos e não tiveram diferença significativa entre si e os tratamentos $3 \mathrm{e} 4$ foram inferiores aos tratamentos 1 e 2 (Figura 6).
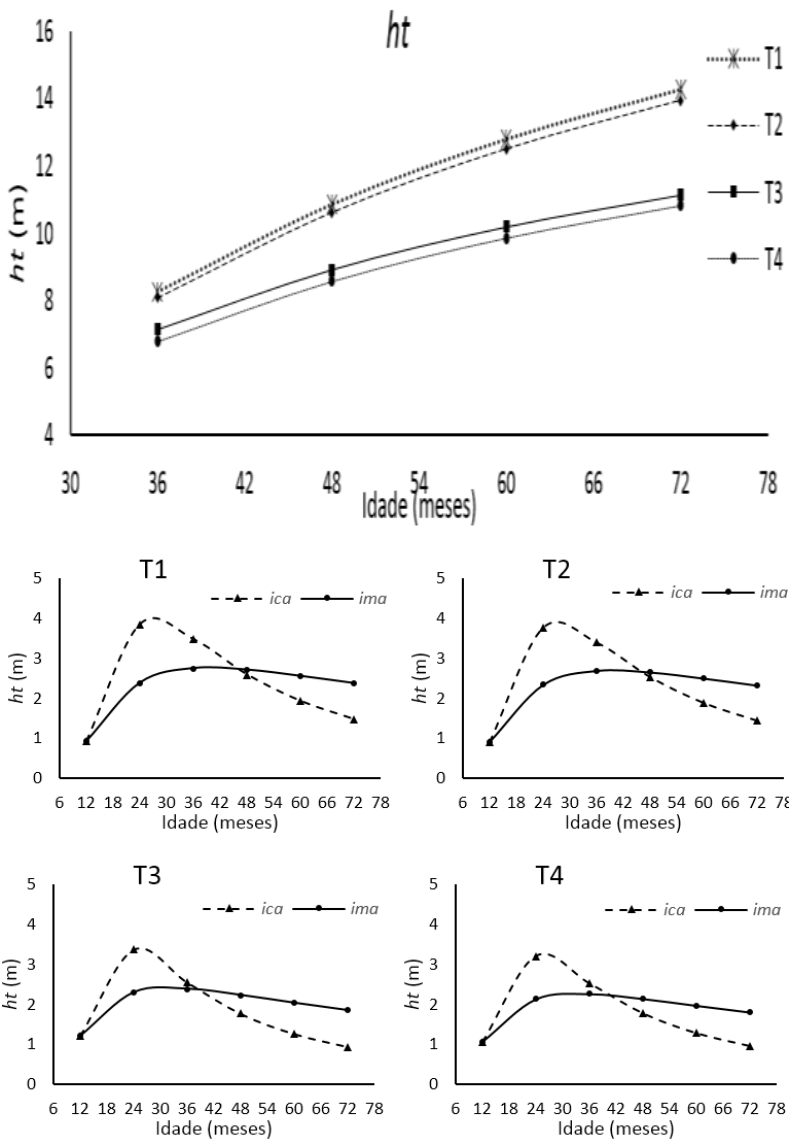

Figura 6. Curvas de crescimento e incremento corrente anual (ica) e incremento médio anual (ima) em altura total $(h t)(\mathrm{m})$ em altura total $(h t)(\mathrm{m})$ de teca para os quatro tratamentos no projeto $\mathrm{B}$.

A área basal $(G)$ dos tratamentos 1 e 2 apresentou os maiores crescimentos (Figura 7). Os tratamentos $3 \mathrm{e} 4$ tiveram os crescimentos em área basal inferiores, não tendo diferença estatística significativa entre si.

$\mathrm{O}$ crescimento em volume total dos tratamentos 1 e 2 foram semelhantes (Figura 8) não diferindo estatisticamente entre si aos 36, 48, 60 e 72 meses. Os tratamentos 3 e 4 tiveram o crescimento inferior aos tratamentos 1 e 2 . 

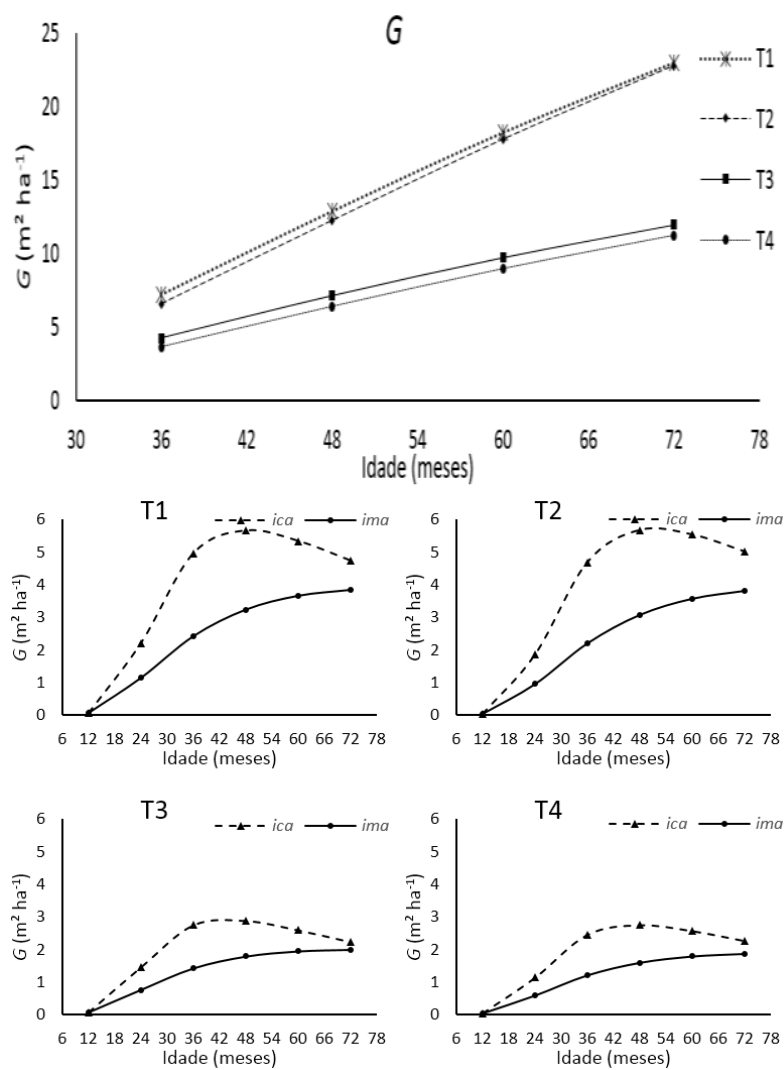

Figura 7. Curvas de crescimento e curvas de incremento corrente anual (ica) e incremento médio anual (ima) em área basal $(\mathrm{G})\left(\mathrm{m}^{2} \mathrm{ha}^{-1}\right)$ de teca para os quatro tratamentos no projeto $\mathrm{B}$.
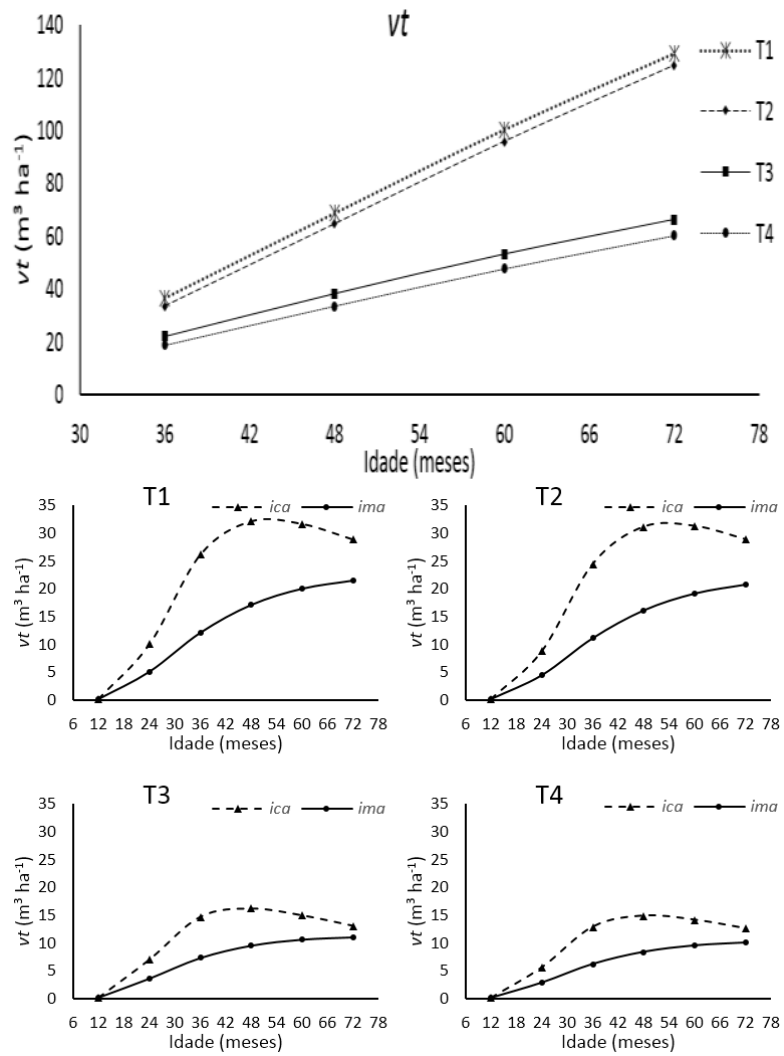

Figura 8. Curvas de crescimento e curvas de incremento corrente anual (ica) e incremento médio anual (ima) em volume total $(v t)\left(\mathrm{m}^{3} \mathrm{ha}^{-1}\right)$ de teca para os quatro tratamentos no projeto $\mathrm{B}$.

\section{Projeto A e Projeto B}

Aos 72 meses do plantio foi realizada uma comparação em porcentagem do volume total da teca entre os projetos A e B (Figura 9).

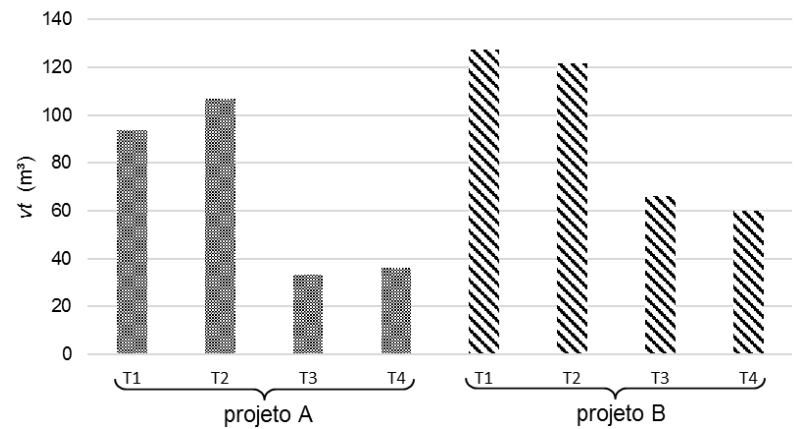

Figura 9. Volume total de teca para o projeto A e projeto B aos 72 meses de idade em sistema agroflorestal em Figueirópolis D’Oeste, MT.

A teca clonal no projeto $\mathrm{B}$, tratamento 1 obteve um volume total $26 \%$ maior em relação a teca clonal no projeto A, tratamento 1 .

A teca clonal no projeto $\mathrm{B}$, tratamento 2 obteve um volume total $12 \%$ maior em relação a teca clonal no projeto A, tratamento 2 .

A teca seminal no projeto $B$, tratamento 3 obteve um volume total $49 \%$ maior em relação a teca seminal no projeto A, tratamento 3 .

A teca seminal no projeto $\mathrm{B}$, tratamento 4 obteve um volume total $40 \%$ maior em relação a teca seminal no projeto A, tratamento 4.

Quando se compara o desenvolvimento da teca no projeto A, tratamento 1 com o projeto B, tratamento 2, observa-se um valor de $23 \%$ menor em volume total. Porém, do ponto de vista econômico, no projeto $\mathrm{A}$, tratamento 1 , o produtor obtém mais dois tipos de renda.

\section{Conclusões}

A teca oriunda de mudas clonais obteve desenvolvimentos superiores em todos os tratamentos avaliados para as variáveis altura total, diâmetro à altura do peito, área basal, volume total e taxa de sobrevivência em todas as idades avaliadas em relação aos tratamentos com teca de origem seminal.

Até aos 72 meses de idade a presença do milho não apresenta influência significativa no desenvolvimento da teca.

\section{Referências}

Campos JCC, Leite HG. Mensuração florestal: perguntas e respostas. 4. ed. Viçosa: UFV, 2013. 605p.

Cruz J. P.; Leite, H. L.; Soares, C. P. B.; Campos, J. C. C.; Smit, L.; Nogueira, G. S. Modelos de crescimento e produção para plantios comerciais jovens de Tectona grandis em Tangará da Serra, Mato Grosso. Revista Árvore, v.32, n.5, p. 821-828, 2008. doi: 10.1590/S010067622008000500006 .

IBÁ - Indústria Brasileira de Árvores. Relatório 2017. Disponível em: <https://www.iba.org/datafiles/publicacoes/pdf/ibarelatorioanual2017.pdf >. 
Khasanah, N.; Perdana, A.; Rahmanullah, A.; Manurung, G.; Roshetko, J. M.; Noordwijk, M. V. Intercropping teak (Tectona grandis) and maize (Zea mays): bioeconomic trade-off analy sis of agroforestry management practices in Gunungkidul, West Java. Agroforestry Sy stems, v. 89, n. 6, p. 1019-1033, 2015. doi: 10.1007 / s 10457-015-9832-8.

Medeiros, R. A.; Paiva, H. N.; Leite, H. G.; Oliveira Neto, S. N.; Vendrúscolo, D. G. S.; SILVA, F. T. Análise silvicultural e econômica de plantios clonais e seminais de Tectona grandis 1.f. em sistema taungya. Revista Árvore, Viçosa-MG, v.39, n.5, p.893-903, 2015. doi: 10.1590/010067622015000500012 .

Midgley S.; Somaiy a R. T.; Stevens P. R.; Brown A.; Kien, N. D.; Laity, R. Planted teak: global production and markets, with reference to Solomon Islands. Australian Centre for International Agricultural Research: Technical Reports No 85, Canberra. 2015. 92p.

Moretti, M. S.; Tsukamoto Filho, A. de A.; Costa, R. B. da; Rondon Neto, R. M.; MEDEIROS, R. A.; Sousa, R. A. T M. Crescimento inicial de plantas de teca em monocultivo e sistema Taungy a com milho em Figueiropolis D'Oeste, Estado de Mato Grosso. Scientia Forestalis, v. 42, n. 102, p. 269-277, 2014.

Ribeiro, F. A.; Macedo, R. L. G.; Venturim, N.; Morais, V. M.; Gomes, J.E.; Yoshitami, M. Jr. Feitos da adubação de plantio sobre o estabelecimento de mudas de Tectona grandis L.f. (teca). Revista Científica Eletrônica de Engenharia Florestal, n. 4, v. 7, p. 1-13. 2006.

Santos-Martin, F. e Van Noordwijk, M. Trade-offs analysis for possible timber-based agroforestry scenarios using native trees in the Philippines. Agroforestry Sy stems, v. 76. P. 555-567. 2009. doi: 10.1007 / s10457-009-9208-z.

Silva, F. R. da; Santos, A. F. A.; Souza, W. C.; Garcia, M. L. Desempenho inicial de Tectona grandis L. f. em sistema silvipastoril na região norte de Mato Grosso, Brasil. Múltiplos olhares sobre a Biodiversidade III. p 153-164, 2014

Silva, R. S. da; Vendruscolo, D. G. S.; Rocha, J. R. M. da; Chaves, A. G. S.; Souza, H. S.; Motta, A. S. da. Desempenho Silvicultural de Tectona grandis L. f. em Diferentes Espaçamentos em Cáceres, MT. Floresta e Ambiente, v. 23 n. 3, 2016. 397-405. doi:10.1590/21798087.143015

Silva, I. C. Sistemas agroflorestais: conceitos e métodos. 1. ed. Itabuna: SBSAF, 2013. 308p.

Tonini, H.; Costa, M. C. G. C.; Schwengber, L. A. M. Crescimento da teca (Tectona grandis) em reflorestamento na Amazônia Setentrional. Pesquisa Florestal Brasileira, n. 59, p. 05-14. 2009. doi: 10.4336/2009.pfb.59.05.

Xavier, A; Silva, R. L. Evolução da silvicultura clonal de Eucaly ptus no Brasil. Agronomía Costarricense 34(1): 93 98. ISSN:0377-9424 / 2010

Xavier, A.; Wendling, I; Silva, L. S. Silvicultura clonal: princípios e técnicas. Viçosa: UFV, 2009. 272 p. 